\title{
Primary Assessment and Care in Maxillofacial Trauma
}

\author{
Saurabh Saigal and Manal M. Khan
}

\subsection{Introduction $[1,2]$}

Maxillofacial injuries are common in polytrauma patients, and spectrum can be minor to life-threatening injuries. Primary assessment and management can be very important. They can involve facial soft tissues, facial bones, or both. Maxillofacial injuries can be due to various causes including road traffic accidents, assaults, and fall from height, industrial injuries, animal bites, sports injuries, burns, and war injuries. Lifethreatening maxillofacial injuries can complicate the initial management of a trauma patient due to presence of concomitant injuries to airway, head, or cervical spine.

The mechanism for this injury is exemplified by an unbelted automobile passenger who is thrown into the windshield and dashboard. Trauma to midface can produce fractures and dislocations that compromise the nasopharynx and oropharynx. Facial fractures can be associated with hemorrhage, increased secretion, and dislodged teeth. Fractures of mandible, especially bilateral body fractures, can cause loss of normal airway and structural support. Airway obstruction can result if the patient is in supine position.

The term "golden hour" has to be kept in mind while handling the trauma patient which indicates that the injured patient has $1 \mathrm{~h}(60 \mathrm{~min})$ from the time of injury to receive definitive care. After that there will be significant increase in the morbidity and mortality of the patient. "Chances of survival of the critically injured patient will largerly determine after the first hour" [3].

Electronic Supplementary Material The online version of this chapter (https://doi.org/10.1007/978-981-15-1346-6_48) contains supplementary material, which is available to authorized users.

S. Saigal $(\bowtie)$

Department of Anaesthesiology, AIIMS Bhopal,

Bhopal, Madhya Pradesh, India

e-mail: saurabh.criticalcare@aiimsbhopal.edu.in

\section{M. Khan}

Department of Burns and Plastic Surgery, AIIMS Bhopal,

Bhopal, Madhya Pradesh, India

\subsection{Triage $[4]$}

Sorting of patients based on their need for treatment and the available resources to provide the treatment is triage. This sorting may be carried out by the paramedic team at the accident scene or receiving hospital who decides what level of care is required. It may be based on which patients need immediate, lifesaving interventions, which can wait and which are, in fact, beyond saving. Depending on the urgency of treatment required, maxillofacial injuries can be broadly placed into one of four groups (Table 48.1).

Appropriate triage and prompt evaluation, using the Advanced Trauma Life Support (ATLS) system, benefit patients [5].

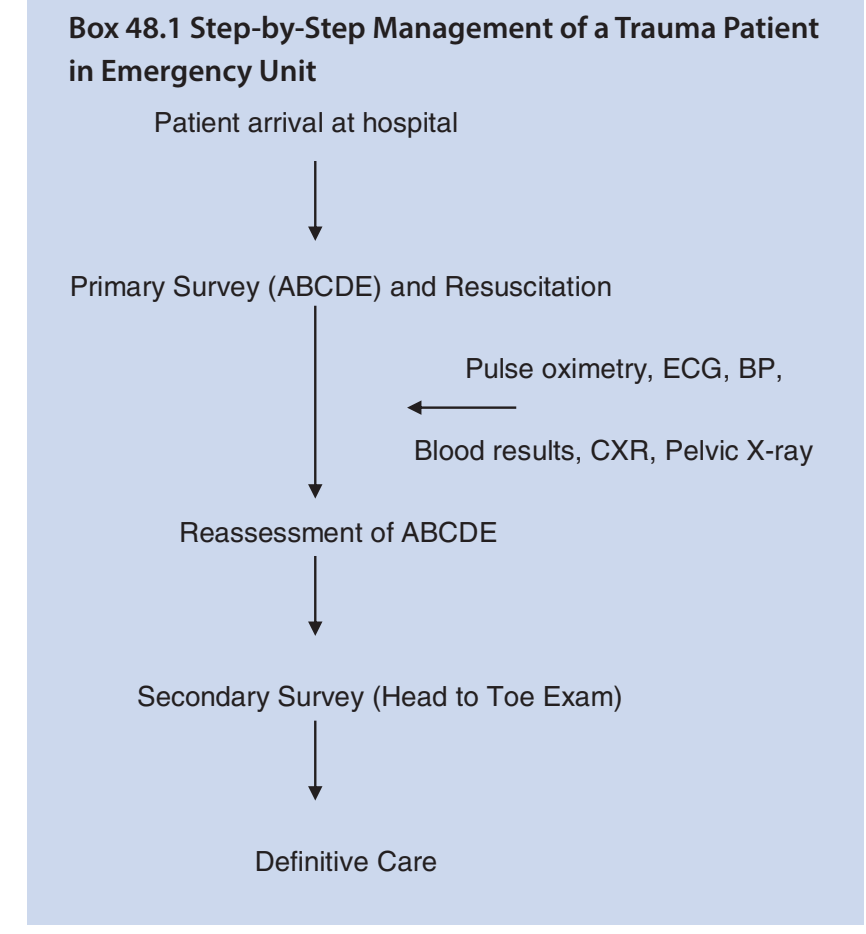

Box 48.1 Step-by-Step Management of a Trauma Patient rgency Unit 
Table 48.1 Triage color coding

\begin{tabular}{|l|l|l|}
\hline Group & Color coding & $\begin{array}{l}\text { Description } \\
\text { Intervention required within few minutes. } \\
\text { Immediate lifesaving management needed } \\
\text { (airway compromise, severe uncontrolled } \\
\text { blood loss) }\end{array}$ \\
\hline Group 2 & Yellow & $\begin{array}{l}\text { Intervention required within few hours. } \\
\text { Patient stable but urgent management } \\
\text { needed (cranial fractures with open dura, } \\
\text { contaminated wounds, and open fractures) }\end{array}$ \\
\hline Group 3 & Green & $\begin{array}{l}\text { Intervention required within few days. } \\
\text { Can wait a day or two (clean laceration } \\
\text { and some fractures) }\end{array}$ \\
\hline Group 4 & Black & $\begin{array}{l}\text { Intervention required within few weeks. } \\
\text { Can wait for more days (most fractures) }\end{array}$ \\
\hline
\end{tabular}

\subsubsection{Glasgow Coma Scale (Please Refer Table 7.5 in Chap. 7 of this book)}

There are three variables used with the scale:

Best motor response (level of central nervous system function) Best verbal response (ability of CNS to integrate information) Eye opening (brainstem activity)

\subsection{Primary Survey and Resuscitation [6]}

The primary survey is a rapid, reproducible physical examination to evaluate every trauma patient and is designed to diagnose and treat immediately life-threatening conditions first. All patients are evaluated for physiologic or anatomic derangements that could lead to early mortality and morbidity. Treatment of problems identified during the primary survey begins without delay, before the survey is completed.

The sequence of primary survey is $\mathrm{ABCDE}$ :

1. Airway with cervical spine control

2. Breathing with ventilation

3. Circulation and hemorrhagic control

4. Disability management (neurological)

5. Exposure with environment control

Patient monitoring (pulse oximetry, electrocardiogram (ECG), blood pressure (BP), blood results, chest and pelvic radiographs) should run in parallel with the primary survey. Team approach is crucial for primary survey.

\subsubsection{Airway with Cervical Spine Control}

Airway assessment is first and foremost priority of primary survey. Patients with maxillofacial and head injuries are at high risk of compromised airway.

Causes of upper airway obstruction

1. Foreign body aspiration

2. Tongue fall position

3. Tracheal, laryngeal, facial, and mandibular fractures

4. Regurgitation of stomach contents

Maxillofacial injuries can cause airway obstruction because of:

- Bilateral anterior mandible fracture allows the tongue to fall posteriorly

- Downward and backward displacement of maxilla into nasopharynx, after midface fracture

- Blood clots and secretions

- Foreign bodies in the form of avulsed teeth or dentures

The chin lift or jaw-thrust maneuver is recommended to achieve patency of airway. The patient's head and neck should not be hyperextended, hyperflexed, or rotated to establish and maintain the airway. There may be associated cervical spine injury; all the cases are treated as they are having cervical spine injury unless diagnosed otherwise. Hence, cervical spine stabilization should be done.

\subsubsection{Airway and Ventilatory Problems in Maxillofacial Trauma (Please Refer Chap. 7 of this book)}

Early preventable deaths from airway problem after maxillofacial trauma often result from:

- Failure to identify the need for an airway intervention

- Inability to establish an airway

- Lack of backup or alternative airway plan in the setting of failed intubation attempts 
- Failure to recognize an incorrectly placed airway

- Displacement of a previously established airway

- Failure to identify the need for ventilation

- Aspiration of gastric contents during dealing with airway

\subsubsection{Airway and Ventilation Are the First Priorities}

Airway compromise may be sudden and complete, insidious and partial, and/or progressive and recurrent. The early sign of airway or ventilator compromise is tachypnea or inability to speak words or sentences. "Talking patient" provides reassurance for that period of time that the airway is patent and not compromised. Failure to respond or an inappropriate response suggests an altered level of consciousness, airway and ventilator compromise, or both. Patient with the altered level of consciousness is at particular risk for airway compromise and aspiration, so he requires definite airway.

\subsubsection{Objective Signs of Airway Obstruction}

1. Observe the patient for agitation and obtundation, suggest hypercarbia, and cyanosis indicates hypoxemia due to inadequate oxygenation which can be identified by inspection of nail beds and circum-oral skin. However, cyanosis is a late finding of hypoxia. Pulse oximetry is used early in the airway assessment, to detect inadequate oxygenation prior to development of cyanosis. Look for retractions and use of accessory muscles of ventilation that, when present, provide additional evidence of airway compromise.

2. Listen for abnormal, noisy sound breathing which is usually snoring, gurgling, and crowing sound (stridor) and can be associated with partial obstruction of the pharynx or larynx. Hoarseness (dysphonia) implies functional, laryngeal obstruction.

3. Feel for location of trachea, and quickly determine whether it is in the midline position.

4. Evaluate patient behavior. Abusive and belligerent patient may in fact have hypoxia and should not be presumed to be intoxicated (suggestive of hypoxia, then only presume intoxication).

\subsubsection{Airway Management $[7,8]$}

To assess airway patency and adequate ventilation quickly and accurately, pulse oximetry and end-tidal $\mathrm{CO}_{2}$ measurement are essential. There are some measures to improve the oxygenation including airway maintenance techniques, definitive airway measures, or surgical airways. Because abovementioned measures include some movement of the neck, it is important to maintain cervical spine protection in all patients of trauma.
High-flow oxygen is required both before and immediately after airway management measures are instituted. A rigid suction device is essential and should be readily available. Nasal route for endotracheal route should not be chosen in patients with facial injury and midface injury. Patients who are wearing helmet and require airway management need their head and neck held in a neutral position. For this two-person procedure, one person provides manual inline stabilization from below, while the second person expands the helmet laterally and removes it from above. Then inline stabilization is re-established from above. And patient's head and neck are secured during airway management. Removal of the helmet using a cast cutter while stabilizing the head and neck can minimize cervical spine ( $\mathrm{C}$ spine) motion in patients with known $\mathrm{C}$ spine injury [9].

Tracheostomy - Video 48.1.

\section{Box 48.2 Need for Airway Protection}

1. Severe maxillofacial fractures

2. Risk for obstruction

- Neck hematoma

- Laryngeal or trachea

- Stridor

3. Risk for aspiration

(i) Bleeding

(ii) Vomiting

4. Unconscious

\section{Box 48.3 Need for Ventilation or Oxygenation}

1. Inadequate respiratory efforts

- Tachypnea

- Hypoxia

- Hypercapnia

- Cyanosis

2. Massive blood loss and need for volume resuscitation

3. Severe closed injury with need for brief hyperventilation if acute neurologic deterioration occurs

4. Apnea

- Neuromuscular paralysis

- Unconscious

\subsubsection{Breathing with Ventilation}

Next, the patient's breathing, ventilation, and oxygenation should be assessed, and any life-threatening derangements must be treated. Physical examination, pulse oximetry, and continuous end-tidal carbon dioxide monitoring should be used. For proper ventilation the lungs, chest wall, and diaphragm must all function adequately. 
The most common interventions performed during the primary survey to support breathing are supplemental oxygen delivery, assisted or mechanical ventilation, and tube thoracotomy or chest tube insertion.

\subsubsection{Ventilation}

Sometimes it will happen that airway of the patient will be patent but ventilation will be inadequate, so look for the objective signs of inadequate ventilation. Ventilation may be compromised by airway obstruction, altered ventilatory mechanics, and central nervous system depression.

Following are the conditions where the ventilation may be compromised:

1. Direct trauma to the chest, like rib fractures, leading to severe pain during breathing and leads to shallow breathing and hypoxemia.

2. Elderly patients and other individual with pulmonary dysfunction are at significant risk for ventilator failure.

3. Intracranial injury can cause abnormal breathing patterns and compromised adequacy of ventilation.

4. Cervical spinal cord injury can result in diaphragmatic breathing and interfere with the ability to meet increased oxygen demands.

5. Complete cervical cord transection, which spares the phrenic and results in abdominal breathing paralysis of the intercostal muscles, and assisted ventilation may be required.

\subsubsection{Objective Signs of Inadequate Ventilation}

1. Symmetrical rise and fall of the chest and adequate chest wall excursion indicate the adequate ventilation, but asymmetrical rise and fall suggests splitting of rib cage or flail chest. Labored breathing may indicate an imminent threat to the patient's ventilation.

2. Listen for movement of air on both sides of the chest. Decreased or absent sounds over one or both hemithoraces should alert examiner to the presence of thoracic injury. Beware of rapid respiratory rate-tachypnea can indicate respiratory distress.

3. Use a pulse oximeter: This device provides information regarding patient's oxygen saturation and peripheral perfusion.

\subsubsection{Circulation and Hemorrhage Control}

Circulation must be assessed to determine the presence or absence of shock after addressing the highest priorities in the primary survey (airway and breathing). Shock is defined as inadequate organ perfusion and tissue oxygenation. In the trauma patient, shock is assumed to be hypovolemic/hemorrhagic, and resuscitation begins as soon as vascular access can be obtained. The possibility of neurogenic shock (e.g., spinal cord injury) or cardiogenic shock (e.g., pericardial tamponade) should also be considered. The focus of this segment of the primary survey should be assessing for the presence of shock, determining the cause (usually blood loss), and beginning resuscitation.

Signs of poor perfusion include a weak pulse, cool or clammy extremities, dry mucous membranes, pale skin, and confusion. A normal mental status examination confirms the presence of acceptable cerebral perfusion. The goal of resuscitation is to maintain tissue perfusion and homeostasis.

\subsubsection{Bleeding}

External or internal bleeding source should be identified. External hemorrhage is identified and controlled during the primary survey. Rapid external blood loss is managed:

- By direct manual pressure on the wound

- Tourniquets which are effective in massive exsanguinations from an extremity

Internal hemorrhage areas are chest, abdomen, retroperitoneum, pelvis, and long bones. Chest X-ray, pelvic X-ray, or focused assessment sonography in trauma (FAST) can be done to identify the source of bleeding.

\subsubsection{Disability Management}

The primary focus is on rapidly determining a patient's mental status and neurologic function via physical exami- 
nation. The Glasgow Coma Scale (GCS) is a rapid and reliable way to quantify a patient's level of consciousness (Table 7.5). The GCS score allows for quick communication among clinicians about a patient's current mental status and can be important for decision-making. The neurologic assessment also includes an examination of the cranial nerves, pupils, and sensory and motor function.

\subsubsection{Exposure with Environment Control}

Exposure and environment are the final components of the primary survey. While lowering priority, they are still vital to the successful management of the trauma patient. The patients should be completely exposed so that injuries can be fully assessed. Decontamination may also be needed, depending on the nature of the trauma. Protection from hypothermia and continuous temperature monitoring are essential.

\subsubsection{Monitoring of Adequacy of Oxygenation}

Oxygenated inspired air is best provided via tight-fitting oxygen reservoir face mask with a flow rate of at least $11 \mathrm{~L} /$ min. Other methods (e.g., nasal catheter and non-breather mask) can improve the inspired oxygen concentration. Pulse oximetry is a noninvasive method of continuously measuring the oxygen saturation $\left(\mathrm{O}_{2}\right.$ sat $)$ of arterial blood. It does not measure the partial pressure of oxygen $\left(\mathrm{PaO}_{2}\right)$, and depending upon the position of oxy-hemoglobin dissociation curve, the $\mathrm{PaO}_{2}$ can vary widely. However a measured saturation of $95 \%$ or greater by pulse oximetry strongly suggests adequate peripheral artery oxygenation $\left(\mathrm{PaO}_{2}>70 \mathrm{mmHg}\right)$.

Pulse oximetry requires intact peripheral perfusion and can't distinguish oxy-hemoglobin from carboxy-hemoglobin or methemoglobin, which limits its usefulness in patients with severe vasoconstriction and those with carbon monoxide poisoning. Profound anemia $(\mathrm{Hb}>5 \mathrm{~g} / \mathrm{dL})$ and hypothermia $\left(<30^{\circ} \mathrm{C}\right)$ decrease the reliability of the technique. However, in most patient pulse oximetry is useful as the continuous monitoring of oxygen saturation provides an immediate assessment of therapeutic intervention.

\subsubsection{Electrocardiographic (ECG) Monitoring}

ECG monitoring of trauma patient is important.

\begin{tabular}{l|l|}
\hline Box 48.4 & \\
\hline Injury indication & ECG changes \\
\hline Blunt cardiac injury & $\begin{array}{l}\text { Dysrhythmias including unexplained } \\
\text { tachycardia, atrial fibrillation, } \\
\text { premature ventricular contractions, } \\
\text { and ST segment changes }\end{array}$ \\
\hline $\begin{array}{l}\text { Cardiac tamponade } \\
\text { Tension pneumothorax } \\
\text { Profound hypovolemia }\end{array}$ & Pulseless electrical activity (PEA) \\
\hline
\end{tabular}

\subsubsection{Fluid Resuscitation}

Warm resuscitation fluids should be given. IV fluid therapy with crystalloids should be initiated. A bolus of 1-2 L of an isotonic solution may be required to achieve an appropriate response in adult patient.

\subsubsection{Urinary and Gastric Catheters}

Urine output monitoring is important to manage the fluid therapy. Urine output reflects renal perfusion, and it is accomplished by insertion of indwelling bladder catheter.

Contraindications to the placement of Foley catheter and urethral injury should be suspected in the presence of:

1. Blood at the urethral meatus

2. Hemorrhage into the scrotum

3. High-riding prostate

A gastric tube is indicated to:

1. Reduce stomach distension

2. Decrease risk of aspiration

3. Assess for upper gastrointestinal hemorrhage from trauma

\subsection{Secondary Survey $[10-12]$}

Once the primary survey is completed, secondary survey is started. If additional personnel is available, then part of secondary survey can be conducted along with primary sur- 
vey; however, it should be made sure that it does not interfere with primary survey, as primary survey is the first priority.

Secondary survey is a complete head to toe examination of the trauma patient which includes complete history taking and physical examination. During secondary survey, a complete neurologic examination is conducted. Patient's GCS score is identified, radiographs are obtained, and laboratory studies are conducted as indicated.

\subsubsection{History Taking}

A complete medical assessment includes history taking and understanding the mechanism of the injury. Often, patient is not in a condition to provide this history. In such cases, it should be obtained from the family members. The history taking involves identification of various allergies, present medications, past history of any illness, pregnancy, last meal taken, and the event of trauma. The mechanism of injury must be understood whether it's inflicted by a penetrating or blunt trauma or if the injury is thermal injury or due to hazardous environment.

\subsubsection{Physical Examination}

During physical examination in secondary survey, a sequence has to be followed, which is examination of head, maxillofacial structures, cervical spine and neck, chest, abdomen, perineum/rectum/vagina, musculoskeletal system, and neurologic system.

\subsubsection{Four-Person Logroll}

To examine the patient's back and remove the spine board, at least four persons are required for logrolling:

(a) Person one stands at the patient's head to control the head and $\mathrm{C}$ spine and two persons along the patient's sides to control the body and extremities.

(b) Three persons maintain the alignment of spine, as the patient is rolled.

(c) Fourth person examines the back and removes the board.

(d) Once the board is removed, patient is returned into the supine position while maintaining the alignment of spine.

\subsubsection{Head}

Secondary survey starts with examination of head which involves identification of injuries. The complete scalp should be examined for lacerations, contusions, and evidence of fractures. Other than these injuries, visual acuity, pupillary size, hemorrhage of conjunctiva and/or fundi, and ocular entrapment must be examined. These aspects must be re-evaluated once the periocular edema subsides.

Visual acuity examination must be done by asking the patient to read printed material such as handheld Snellen chart. To exclude ocular entrapment, ocular movement must be checked.

\subsubsection{Classification of Brain Injury}

\begin{tabular}{|l|l|}
\hline Box 48.5 & \\
\hline Severity & GCS score \\
\hline Minor & $13-15$ \\
\hline Moderate & $9-12$ \\
\hline Severe & $3-8$ \\
\hline
\end{tabular}

Intracranial lesions may be classified as diffuse or focal. The focal lesions include epidural hematomas, subdural hematomas, contusions, and intracerebral hematomas.

Signs of skull base fracture:

1. Hematoma or ecchymosis over the mastoid (Battle's sign)

2. CSF otorrhea or rhinorrhea

3. Hemotympanum

4. Subscleral hemorrhage

Computed tomography (CT) scan of the head should be carried out to accurately assess the neurologic injuries and detect mass lesions. CT scan helps in diagnosing:

- Intracranial hemorrhage

- Contusion

- Skull fractures

- Foreign bodies

- Brain shift

- Hydrocephalus

In a comatose patient, motor responses may be elicited by pinching the trapezius muscle or with nail bed or supraorbital pressure. Testing for doll's eye movements (oculocephalic) the caloric test with ice water (oculovestibular) and testing of corneal responses are deferred to a neurosurgeon. 


\subsubsection{Maxillofacial Structures}

Maxillofacial examination includes examination of soft tissue, palpation of all the bony structures, checking of occlusion, and intraoral examination. Trauma to the maxillofacial region not associated with airway obstruction should be treated once the patient is stabilized after the management of life-threatening injuries.

\subsubsection{Cervical Spine and Neck}

Patients with maxillofacial trauma must be presumed to have an unstable cervical spine injury unless it is proven otherwise. The absence of any neurologic deficit does not exclude the presence of any $\mathrm{C}$ spine injury. A complete cervical spine radiographs and $\mathrm{CT}$ scan should be done to evaluate $\mathrm{C}$ spine injury. The patient wearing any kind of helmet must be removed with extreme care.

C spine injuries can result from one or a combination of the following mechanism of injury:

- Flexion

- Axial loading

- Extension

- Rotation

- Lateral bending

- Distraction

A complete examination of neck includes inspection, palpation, and auscultation. Inspection should be done to note any blunt injury over the neck. Carotid arteries should be palpated and auscultated for bruits. Penetrating injuries of neck are potentially fatal. If the wound is deep, they should not be explored in emergency department [13].

A cervical collar can be applied to the patients suspecting the cervical spinal injury. Its role is to immobilize the $\mathrm{C}$ spine. Cervical collars are divided into two groups: soft and rigid collars. Soft collars are generally used for whiplash injury; some surgeons prefer rigid collars. Rigid collars provide excellent immobilization in transverse and sagittal planes compared with soft collars. However patient's comfort is to be taken into consideration in selecting the collars. The Philadelphia collar is available in two pieces having front and back pieces; it is held together by Velcro straps to support the neck [14].

\subsubsection{Chest}

Chest examination includes inspection, palpation, percussion, and auscultation. Inspection of anterior and posterior chest can identify conditions like open pneumothorax and large flail segments. Contusions and hematoma over chest suggest occult injury. Palpation of entire rib cage, clavicle, and sternum helps in diagnosing fractures. Significant injury may present with pain, dyspnea, and hypoxia.

Auscultation of high anterior chest helps in diagnosis of pneumothorax, whereas posterior base auscultation reveals hemothorax. Cardiac tamponade can be identified by the presence of distant heart sounds and decreased pulse pressure. Presence of distended neck veins suggests cardiac tamponade and tension pneumothorax. A chest radiograph may confirm the presence of hemothorax or simple pneumothorax.

\subsubsection{Classification of Chest Trauma}

Immediate life-threatening chest injuries are to be identified and treated during primary survey.

These six life-threatening chest injuries include:

1. Open pneumothorax

2. Tension pneumothorax

3. Open pneumothorax

4. Flail chest and pulmonary contusion

5. Massive hemothorax

6. Cardiac tamponade

\subsubsection{Managing Chest Trauma [15]}

The basic principles of management remain the same with the universal sequence of airway, breathing, and circulation to be treated in that sequence.

\subsubsection{Airway}

It is necessary to recognize and address major injuries affecting the airway during the primary survey. Airway patency and air exchange should be assessed by listening for air movement at the patient's nose, mouth, and lung fields; inspecting the oropharynx for foreign body obstruction; and observing for intercostal and supraclavicular muscle retractions. Laryngeal injury can accompany major thoracic trauma. Although the clinical presentation is occasionally delayed, acute airway obstruction from laryngeal trauma is a life-threatening injury. Injury to the upper chest can create a 
palpable defect in the region of the sternoclavicular joint, with posterior dislocation of the clavicular head, which causes upper airway obstruction. Identification of this injury is made by listening for upper airway obstruction (stridor) or a marked change in the expected voice quality, if the patient is able to talk. Management consists of a closed reduction of the injury, which can be performed by extending the shoulders or grasping the clavicle with a pointed instrument, such as a towel clamp, and manually reducing the fracture. Once reduced, this injury is usually stable if the patient remains in the supine position.

\subsubsection{Breathing}

The patient's chest and neck should be completely exposed to allow for assessment of breathing and the neck veins. This may require temporarily releasing the front of the cervical collar following blunt trauma. In this case, cervical spine immobilization should always be actively maintained by holding the patient's head while the collar is loose. Respiratory movement and quality of respirations are assessed by observing, palpating, and listening. Important, yet often subtle, signs of chest injury or hypoxia include an increased respiratory rate and change in the breathing pattern, which is often manifested by progressively shallower respirations. Cyanosis is a late sign of hypoxia in trauma patients. However, the absence of cyanosis does not necessarily indicate adequate tissue oxygenation or an adequate airway. The major thoracic injuries that affect breathing and that must be recognized and addressed during the primary survey include tension pneumothorax, open pneumothorax (sucking chest wound), flail chest and pulmonary contusion, and massive hemothorax.

Important: After intubation, one of the common reasons for loss of breath sounds in the left thorax is a right mainstem intubation. During the reassessment, be sure to check the position of the endotracheal tube before assuming that the change in physical examination is due to a pneumothorax or hemothorax.

\subsubsection{Tension Pneumothorax}

A tension pneumothorax develops when a "one-way valve" air leak occurs from the lung or through the chest wall. Air is forced into the pleural space without any means of escape, eventually completely collapsing the affected lung. The mediastinum is displaced to the opposite side, decreasing venous return and compressing the opposite lung. Shock results from the marked decrease in venous return causing a reduction in cardiac output and is often classified as obstructive shock (Fig. 48.1). The most common cause of tension pneumothorax is mechanical ventilation with positivepressure ventilation in patients with visceral pleural injury. However, a tension pneumothorax can complicate a simple pneumothorax following penetrating or blunt chest trauma in which a parenchymal lung injury fails to seal or after a mis-

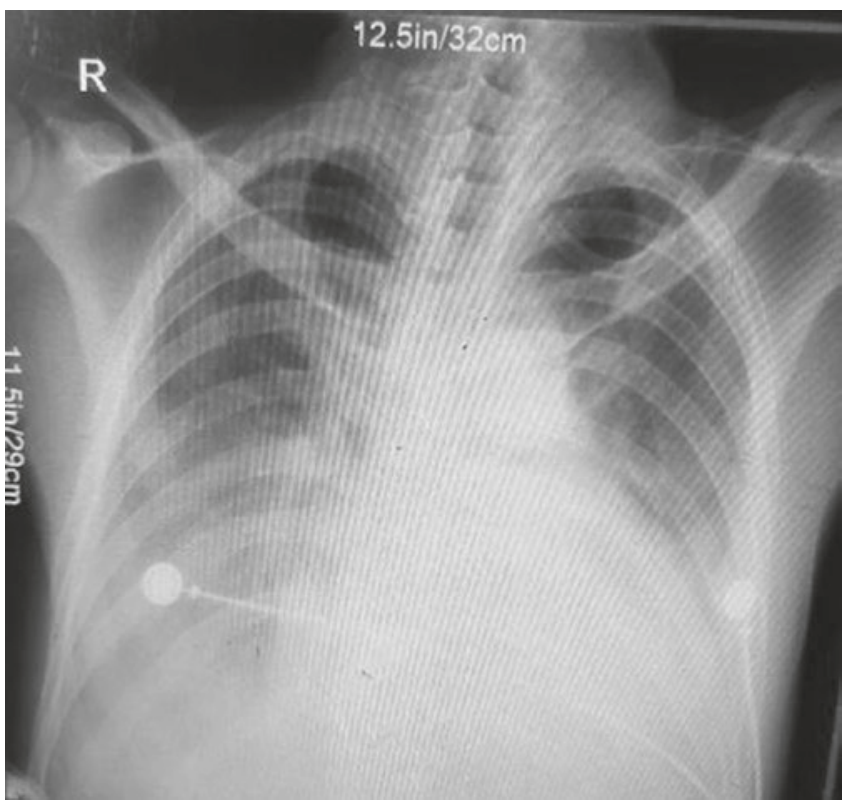

CAssociation of Oral and Maxillofacial Surgeons of India

Fig. 48.1 Tension pneumothorax

guided attempt at subclavian or internal jugular venous catheter insertion. Occasionally, traumatic defects in the chest wall also can cause a tension pneumothorax if incorrectly covered with occlusive dressings or if the defect itself constitutes a flap-valve mechanism. Tension pneumothorax rarely occurs from markedly displaced thoracic spine fractures. Tension pneumothorax is a clinical diagnosis reflecting air under pressure in the affected pleural space. Treatment should not be delayed to wait for radiologic confirmation.

Tension pneumothorax is characterized by some or all of the following signs and symptoms:
Chest pain
Air hunger
Respiratory distress
Tachycardia
Hypotension
Tracheal deviation away from the side of injury
Unilateral absence of breath sounds
Elevated hemithorax without respiratory movement
Neck vein distention
Cyanosis (late manifestation)

Because of the similarity in their signs, tension pneumothorax can be confused initially with cardiac tamponade. Differentiation is made by a hyper-resonant note on percussion, deviated trachea, and absent breath sounds over the 
affected hemithorax, which are signs of tension pneumothorax. Tension pneumothorax requires immediate decompression and may be managed initially by rapidly inserting a large-caliber needle into the second intercostal space in the midclavicular line of the affected hemithorax. However, due to variable thickness of the chest wall, kinking of the catheter, and other technical or anatomic complications, this maneuver may not be successful [16].

When successful, this maneuver converts the injury to a simple pneumothorax; however, the possibility of subsequent pneumothorax as a result of the needle stick now exists, so repeated reassessment of the patient is necessary. Chest wall thickness influences the likelihood of success with needle decompression. Recent evidence suggests that a $5 \mathrm{~cm}$ needle will reach the pleural space $>50 \%$ of the time, whereas an $8 \mathrm{~cm}$ needle will reach the pleural space $>90 \%$ of the time. Even with a needle of the appropriate size, the maneuver will not always be successful. Definitive treatment requires the insertion of a chest tube into the fifth intercostal space (usually at the nipple level), just anterior to the midaxillary line.

In a tension pneumothorax, air from a ruptured lung enters the pleural cavity without a means of escape. As air pressure builds up, the affected lung is compressed, and all of the mediastinal tissues are displaced to the opposite side of the chest.

\subsubsection{Open Pneumothorax (Sucking Chest Wound)}

Large defects of the chest wall that remain open can result in an open pneumothorax, which is also known as a sucking chest wound. Equilibration between intrathoracic pressure and atmospheric pressure is immediate. Air tends to follow the path of least resistance; as such, if the opening in the chest wall is approximately two-thirds of the diameter of the trachea or greater, air passes preferentially through the chest wall defect with each respiratory effort. Effective ventilation is thereby impaired, leading to hypoxia and hypercarbia. Initial management of an open pneumothorax is accomplished by promptly closing the defect with a sterile occlusive dressing. The dressing should be large enough to overlap the wound's edges and then taped securely on three sides in order to provide a flutter-type valve effect. As the patient breathes in, the dressing occludes the wound, preventing air from entering. During exhalation, the open end of the dressing allows air to escape from the pleural space. A chest tube remote from the wound should be placed as soon as possible. Securely taping all edges of the dressing can cause air to accumulate in the thoracic cavity, resulting in a tension pneumothorax unless a chest tube is in place. Any occlusive dressing (e.g., plastic wrap or petrolatum gauze) may be used as a temporary measure so that rapid assessment can continue. Subsequent definitive surgical closure of the defect is frequently required [17].

\subsubsection{Flail Chest and Pulmonary Contusion}

A flail chest occurs when a segment of the chest wall does not have bony continuity with the rest of the thoracic cage. This condition usually results from trauma associated with multiple rib fractures, that is, two or more adjacent ribs fractured in two or more places (Fig. 48.2). The presence of a flail chest segment results in disruption of normal chest wall movement. Although chest wall instability can lead to paradoxical motion of the chest wall during inspiration and expiration, this defect alone does not cause hypoxia. The major difficulty in flail chest stems from the injury to the underlying lung (pulmonary contusion). If the injury to the underlying lung is significant, serious hypoxia can result. Restricted chest wall movement associated with pain and underlying lung injury is major causes of hypoxia. Flail chest may not be apparent initially if a patient's chest wall has been splinted, in which case he or she will move air poorly and movement of the thorax will be asymmetrical and uncoordinated. Palpation of abnormal respiratory motion and crepitation of rib or cartilage fractures can aid the diagnosis. A satisfactory chest $\mathrm{X}$-ray may suggest multiple rib fractures but may not show costochondral separation. Initial treatment of flail chest includes adequate ventilation, administration of humidified oxygen, and fluid resuscitation. In the absence of systemic hypotension, the administration of crystalloid intravenous solutions should be carefully controlled to prevent volume overload, which can further compromise the patient's respiratory status.

The definitive treatment is to ensure adequate oxygenation, administer fluids judiciously, and provide analgesia to improve ventilation. The latter can be achieved with intravenous narcotics or local anesthetic administration, which

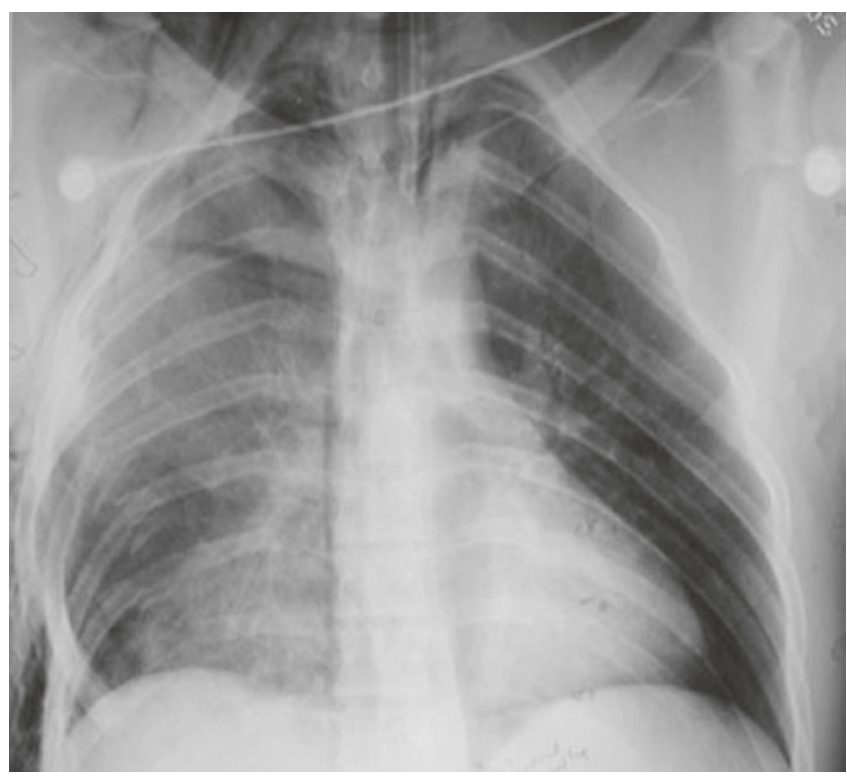

CAssociation of Oral and Maxillofacial Surgeons of India

Fig. 48.2 Flail chest with pulmonary contusion 
avoids the potential respiratory depression common with systemic narcotics. The options for administration of local anesthetics include intermittent intercostal nerve block(s) and intrapleural, extrapleural, or epidural anesthesia. When used properly, local anesthetic agents can provide excellent analgesia and prevent the need for intubation. However, prevention of hypoxia is of paramount importance for trauma patients, and a short period of intubation and ventilation may be necessary until diagnosis of the entire injury pattern is complete. A careful assessment of the respiratory rate, arterial oxygen tension, and work of breathing will indicate appropriate timing for intubation and ventilation [18].

\subsubsection{Massive Hemothorax}

Massive hemothorax results from the rapid accumulation of more than $1500 \mathrm{~mL}$ of blood or one-third or more of the patient's blood volume in the chest cavity (Fig. 48.3). It is most commonly caused by a penetrating wound that disrupts the systemic or hilar vessels. However, massive hemothorax can also result from blunt trauma. In patients with massive hemothorax, the neck veins may be flat as a result of severe hypovolemia, or they may be distended if there is an associated tension pneumothorax. Rarely will the mechanical effects of massive intrathoracic blood shift the mediastinum enough to cause distended neck veins. A massive hemothorax is suggested when shock is associated with the absence of breath sounds or dullness to percussion on one side of the chest. This blood loss is complicated by hypoxia. Massive hemothorax is initially managed by the simultaneous restoration of blood volume and decompression of the chest cavity.

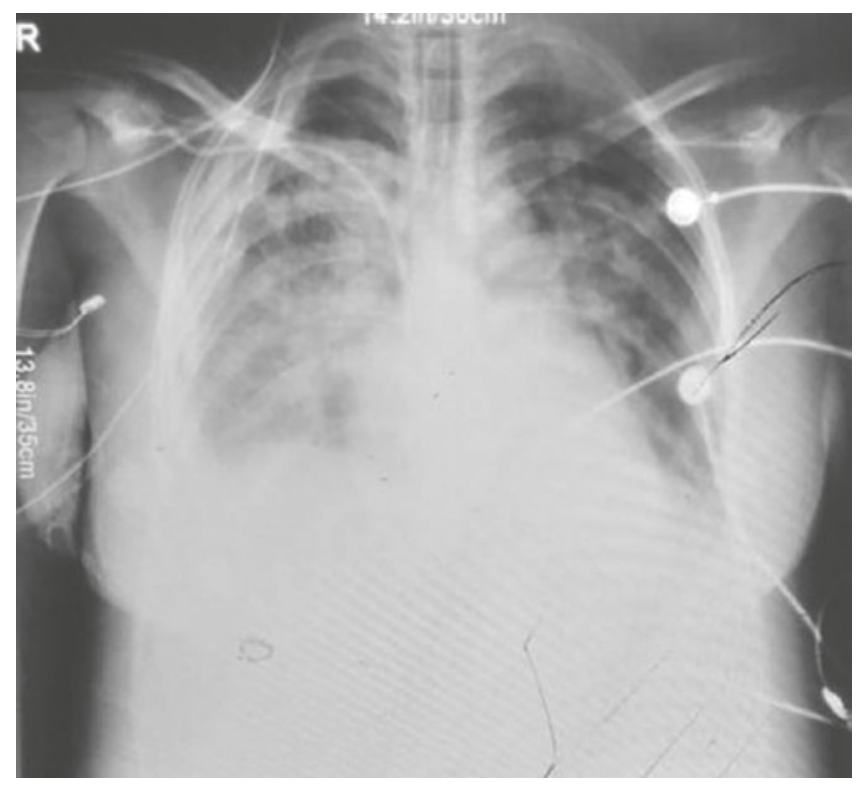

(CAssociation of Oral and Maxillofacial Surgeons of India

Fig. 48.3 Hemothorax
Large-caliber intravenous lines and a rapid crystalloid infusion are begun, and type-specific blood is administered as soon as possible. Blood from the chest tube should be collected in a device suitable for autotransfusion. A single chest tube (36 or 40 French) is inserted, usually at the nipple level, just anterior to the midaxillary line, and rapid restoration of volume continues as decompression of the chest cavity is completed. When massive hemothorax is suspected, prepare for autotransfusion. If $1500 \mathrm{~mL}$ of fluid is immediately evacuated, early thoracotomy is almost always required. Patients who have an initial output of less than $1500 \mathrm{~mL}$ of fluid, but continue to bleed, may also require thoracotomy. This decision is not based solely on the rate of continuing blood loss (200 $\mathrm{mL} / \mathrm{h}$ for $2-4 \mathrm{~h}$ ) but also on the patient's physiologic status. The persistent need for blood transfusions is an indication for thoracotomy. During patient resuscitation, the volume of blood initially drained from the chest tube and the rate of continuing blood loss must be factored into the amount of intravenous fluid required for replacement. The color of the blood (indicating an arterial or venous source) is a poor indicator of the necessity for thoracotomy. Penetrating anterior chest wounds medial to the nipple line and posterior wounds medial to the scapula should alert the practitioner to the possible need for thoracotomy because of potential damage to the great vessels, hilar structures, and the heart, with the associated potential for cardiac tamponade. Thoracotomy is not indicated unless a surgeon, qualified by training and experience, is present.

\subsubsection{Cardiac Tamponade}

Cardiac tamponade (Fig. 48.4) most commonly results from penetrating injuries. However, blunt injury also can cause the pericardium to fill with blood from the heart, great vessels, or pericardial vessel. The human pericardial sac is a fixed fibrous structure; a relatively small amount of blood can restrict cardiac activity and interfere with cardiac filling. Cardiac tamponade may develop slowly, allowing for a less urgent evaluation, or may occur rapidly, requiring rapid diagnosis and treatment. The diagnosis of cardiac tamponade can be difficult in the setting of a busy trauma or emergency room. Cardiac tamponade is indicated by the presence of the classic diagnostic Beck's triad: venous pressure elevation (distended neck veins), decline in arterial blood pressure, and muffled heart sounds. However, muffled heart tones are difficult to assess in the noisy examination area, and distended neck veins may be absent due to hypovolemia. Additionally, tension pneumothorax, particularly on the left side, can mimic cardiac tamponade. Kussmaul's sign (a rise in venous pressure with inspiration when breathing spontaneously) is a true paradoxical venous pressure abnormality associated with tamponade. Pulseless electrical activity (PEA) is suggestive of cardiac tamponade but can have other causes, as listed above [19]. Insertion of a central venous line with measurement of central venous pressure (CVP) may aid diagnosis, but CVP can be elevated for a 


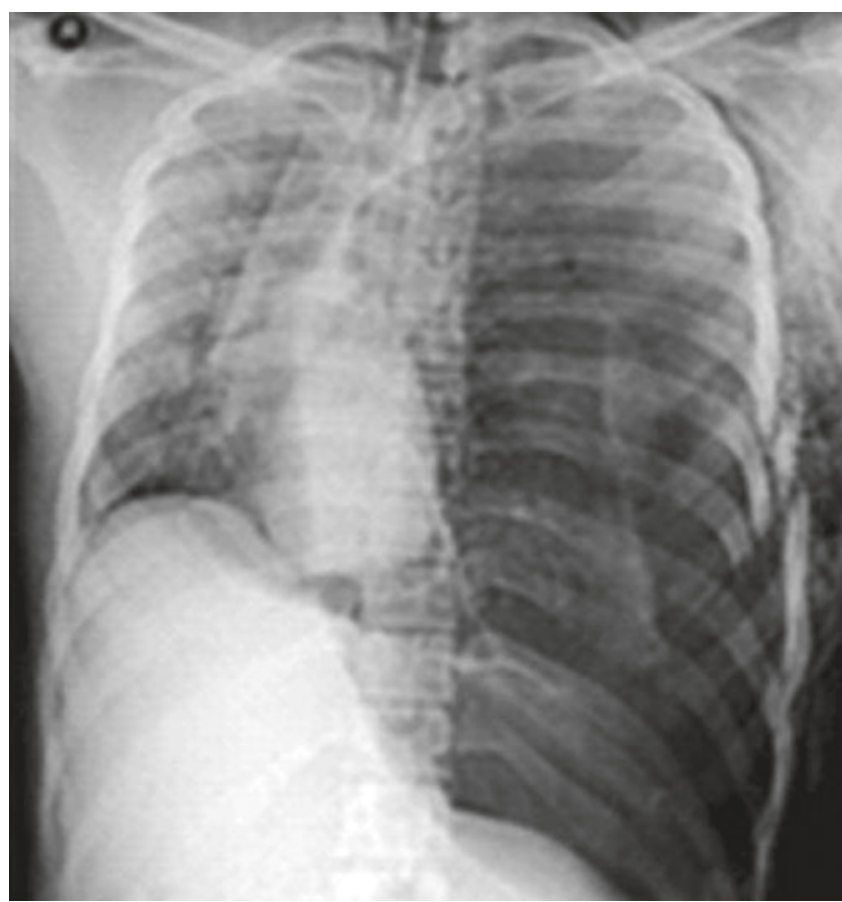

CAssociation of Oral and Maxillofacial Surgeons of India

Fig. 48.4 Cardiac tamponade

variety of reasons. Additional diagnostic methods include echocardiogram, focused assessment sonography in trauma (FAST), or pericardial window. In hemodynamically abnormal patients with blunt or penetrating trauma and suspected cardiac tamponade, an examination of the pericardial sac for the presence of fluid should be obtained as part of a focused ultrasound examination performed by a properly trained provider in the emergency department (ED). FAST is a rapid and accurate method of imaging the heart and pericardium. It is 90-95\% accurate for the presence of pericardial fluid for the experienced operator. Concomitant hemothorax may account for both false-positive and false-negative ultrasound exams. Prompt diagnosis and evacuation of pericardial blood are indicated for patients who do not respond to the usual measures of resuscitation for hemorrhagic shock and in whom cardiac tamponade is suspected. The diagnosis can usually be made with the FAST exam. If a qualified surgeon is present, surgery should be performed to relieve the tamponade. This is best performed in the operating room if the patient's condition allows. If surgical intervention is not possible, pericardiocentesis can be diagnostic as well as therapeutic, but it is not a definitive treatment for cardiac tamponade. However all patients with acute tamponade and a positive pericardiocentesis will require surgery to examine the heart and repair the injury. Pericardiocentesis may not be diagnostic or therapeutic when the blood in the pericardial sac has clotted. Preparation to transfer such a patient to an appropriate facility for defini- tive care is always necessary. Pericardiotomy via thoracotomy is indicated only when a qualified surgeon is available.

\subsubsection{Abdomen $[20]$}

Abdominal injuries must be identified and managed aggressively. It is not as important to arrive at a specific diagnosis as it is to recognize the injury which requires a surgical intervention. It is important to closely observe and re-evaluate the abdomen to identify the blunt injury. The safest management of penetrating wounds is a laparotomy.

\begin{tabular}{|c|c|}
\hline \multicolumn{2}{|l|}{ Abdominal Trauma } \\
\hline Blunt trauma & Penetrating trauma [21] \\
\hline $\begin{array}{l}\text { Direct blow, e.g., } \\
\text { crushing injury to } \\
\text { abdominal viscera and } \\
\text { pelvis }\end{array}$ & $\begin{array}{l}\text { Stab wounds commonly involve liver } \\
(40 \%) \text {, small bowel }(30 \%) \text {, diaphragm } \\
(20 \%) \text {, and colon }(15 \%)\end{array}$ \\
\hline $\begin{array}{l}\text { Shearing injuries, e.g., } \\
\text { laceration of the liver } \\
\text { and spleen }\end{array}$ & $\begin{array}{l}\text { Gunshot wounds commonly involve } \\
\text { small bowel }(50 \%) \text {, colon }(40 \%) \text {, liver } \\
(30 \%) \text {, and abdominal vascular structures } \\
(25 \%)\end{array}$ \\
\hline
\end{tabular}

The diagnostic peritoneal lavage (DPL) was used to diagnose blunt and occasionally penetrating abdominal trauma, but its use was decreased with the advancement of CT and USG. Focused assessment sonography in trauma (FAST) is very helpful to detect the presence of hemoperitoneum.

\subsubsection{Focused Assessment Sonography in Trauma (FAST) [22]}

The ultrasound machine and water-based gel are necessary to perform FAST.

Fast includes the following views:

1. Pericardial view: fluid within the heart should be black.

2. Right upper quadrant view (RUQ): diaphragm-liver interface and Morison's pouch.

3. Left upper quadrant view (LUQ): diaphragm-spleen interface and spleen-kidney interface

4. Suprapubic view: to see bladder

\subsubsection{Perineum/Rectum/Vagina}

Contusions, hematomas, lacerations, and urethral bleedings should be examined in the perineal area. The presence of blood at urethral meatus suggests urethral injury. Inspect the scrotum and perineum for ecchymosis or hematoma, also suggestive of injury to the urethra. In patients with perineal hematoma or high-riding prostate, Foley catheters should be avoided.

Rectal examination can be done to evaluate the presence of blood within the bowel lumen. In patients with the risk of 
vaginal injury, vaginal examination should be carried out. Pelvic compressions should be carried out to identify any pelvic fractures. Pelvic fractures can be suspected by the presence of ecchymosis over iliac wings, pubis, scrotum, or labia. The pelvic fracture can be divided into closed, open book, and vertical shear fracture. Hemorrhage control and fluid resuscitation will be in the initial management of major pelvic disruption associated with hemorrhage. Pelvic binder or sheet can apply sufficient stability for the unstable pelvis at the level of greater trochanters of the femur bone.

\subsubsection{Musculoskeletal System}

The patient must be completely undressed for adequate examination. Extremities should be inspected for deformities, abrasion, and contusions. Presence of tenderness on palpation of bones and abnormal movement helps in identification of occult fractures. Significant injuries of extremities can exist without fracture being evident. Ligament ruptures and a muscle tendon unit injury interferes with active motions. The musculoskeletal examination is incomplete without examination of the back of the patient.

Musculoskeletal injuries are a potential source of blood loss in patients with hemodynamic abnormalities. The proper splint application helps in blood loss control, reducing pain, and preventing further soft tissue injury. Splinting is required in the patients with joint dislocations.

Crush syndrome: it refers to the injured muscle which if left untreated can lead to acute renal failure. Traumatic rhabdomyolysis ranges from asymptomatic illness with elevation of creatine kinase level to a life-threatening condition associated with acute renal failure and disseminated intravascular coagulation (DIC). Early and aggressive fluid therapy protects the kidneys and prevents renal failure in patients with rhabdomyolysis.

Compartment syndrome: develops when the pressure within an osteofascial compartment of muscle causes ischemia and subsequent necrosis. Sometimes fasciotomy is required to manage the condition.

\subsection{Conclusion}

Management of airway is challenging in patients with maxillofacial trauma. Securing airway in these patients is dependent on the clinical status and the features of trauma. Before initiation of airway management, a series of steps are to be planned. For optimal care, one must have adequate knowledge of the specific attributes of difficult airway. Also, the expertise in techniques for managing the difficult airway, familiarity with devices, and recognition of failed airway are necessary. A multidisciplinary approach involving anesthesiologist, maxillofacial surgeon, and a trauma expert must be a practice for better outcome (please refer Chap. 7 for further reading).

\section{References}

1. Perry M. Advanced trauma life support (ATLS) and facial trauma: can one size fit all? Part 1: dilemmas in the management of the multiply injured patient with coexisting facial injuries. Int J Oral Maxillofac Surg. 2008;37:209-14.

2. Perry M, Morris C. Advanced trauma life support (ATLS) and facial trauma: can one size fit all? Part 2: ATLS, maxillofacial injuries and airway management dilemmas. Int J Oral Maxillofac Surg. 2008;37:309-20.

3. Cowley RA. A total emergency medical system for the state of Maryland. Md State Med J. 1975;45:37-45.

4. Johnson G. Trauma triage and trauma system performance. West J Emerg Med. 2016;17:331-2.

5. Ray JM, Cestero RF. Initial management of the trauma patient. Atlas Oral Maxillofac Surg Clin North Am. 2013;21(1):1-7.

6. Gondek S, Schroeder ME, Sarani B. Assessment and resuscitation in trauma management. Surg Clin North Am. 2017;97:985-98.

7. Kovacs G, Sowers N. Airway management in trauma. Emerg Med Clin North Am. 2018;36:61-84.

8. Barak M, Bahouth H, Leiser Y, Abu El-Naaj I. Airway management of the patient with maxillofacial trauma: review of the literature and suggested clinical approach. Biomed Res Int. 2015:724-32.

9. Roccia F, Cassarino E, Boccaletti R, Stura G. Cervical spine fractures associated with maxillofacial trauma: an 11-year review. J Craniofac Surg. 2007;18:1259-63.

10. Galvagno SM, Nahmias JT, Young DA. Advanced Trauma Life Support@ Update 2019: Management and applications for adults and special populations. Anesthesiol Clin. 2019;37:13-32.

11. Sidwell R, Matar MM, Sakran JV. Trauma education and prevention. Surg Clin North Am. 2017;97:1185-97.

12. Rodrigues IFDC. To log-roll or not to log-roll-That is the question! A review of the use of the log-roll for patients with pelvic fractures. Int J Orthop Trauma Nurs. 2017;27:36-40.

13. Rogers WA. Fractures and dislocations of the cervical spine: An end-result study. J Bone Joint Surg Am. 1957;39-A:341-76.

14. Whitcroft KL, Massouh L, Amirfeyz R, Bannister GC. A comparison of neck movement in the soft cervical collar and rigid cervical brace in healthy subjects. J Manipulative Physiol Ther. 2011;34:119-22.

15. Saillant NN, Sein V. Management of severe chest wall trauma. J Emerg Crit Care Med. 2018;2:41.

16. Eckstein M, Suyehara D. Needle thoracostomy in the prehospital setting. Prehosp Emerg Care. 1998;2:132-5.

17. Butler FK, et al. Management of open pneumothorax in tactical combat casualty care: TCCC guidelines change 13-02. J Spec Oper Med. 2013;13:81-6.

18. Pettiford BL, et al. The management of flail chest. Thorac Surg Clin. 2007; 17:25-33. 
19. Spodick DH. Pathophysiology of cardiac tamponade. Chest. 1998;113:1372-8.

20. Matar ZS. The clinical profile of poly trauma and management of abdominal trauma in a general hospital in the central region of the Kingdom of Saudi Arabia. Internet J Surg. 2008;14:11.
21. Demetriades D, et al. The management of penetrating injuries of the back: a prospective study of 230 patients. Ann Surg. 1988;207:72-4.

22. Chaudhry R, Galagali A, Narayanan RV. Focused abdominal sonography in trauma (FAST). Med J Armed Forces India. 2007;63:62-3.

Open Access This chapter is licensed under the terms of the Creative Commons Attribution 4.0 International License (http://creativecommons. org/licenses/by/4.0/), which permits use, sharing, adaptation, distribution and reproduction in any medium or format, as long as you give appropriate credit to the original author(s) and the source, provide a link to the Creative Commons license and indicate if changes were made.

The images or other third party material in this chapter are included in the chapter's Creative Commons license, unless indicated otherwise in a credit line to the material. If material is not included in the chapter's Creative Commons license and your intended use is not permitted by statutory regulation or exceeds the permitted use, you will need to obtain permission directly from the copyright holder. 